\title{
A Compact Frequency Reconfigurable Hybrid DRA for LTE/Wimax Applications
}

\author{
Sajid Aqeel, ${ }^{1}$ M. R. Kamarudin, ${ }^{2}$ Aftab Ahmad Khan, ${ }^{1}$ Jawad Saleem, ${ }^{1}$ Jamal Nasir, \\ Jalil-ur-Rehman Kazim, ${ }^{1}$ and Owais Owais ${ }^{1}$ \\ ${ }^{1}$ Comsats Institute of IT, Abbottabad, Pakistan \\ ${ }^{2}$ Centre for Electronic Warfare, Information and Cyber (EWIC), Cranfield Defence and Security, Cranfield University, \\ Defence Academy of the United Kingdom, Shrivenham SN6 8LA, UK \\ Correspondence should be addressed to Sajid Aqeel; sajidaqeel@ciit.net.pk
}

Received 3 March 2017; Revised 11 May 2017; Accepted 30 May 2017; Published 25 September 2017

Academic Editor: María Elena de Cos Gómez

Copyright (C) 2017 Sajid Aqeel et al. This is an open access article distributed under the Creative Commons Attribution License, which permits unrestricted use, distribution, and reproduction in any medium, provided the original work is properly cited.

\begin{abstract}
A compact hybrid antenna structure with frequency reconfiguration capabilities is presented in this article. The proposed design employs a combination of a rectangular DR element and a coupling slot on the structure's ground plane (GP). The slot shifts the fundamental DRA mode by introducing a slow wave effect and its resonant behavior helps to achieve an omnidirectional pattern at low frequencies. The slot is loaded with a series of PIN diode switches whose ON/OFF combinations alter the effective slot length. Slot loading with PIN diodes results in frequency reconfiguration of the proposed structure with a large tuning range of $76.2 \%$ (between 1.73 and $3.86 \mathrm{GHz}$ ). A parametric analysis was performed to investigate the effects of slot length, width, and position on the reflection coefficients of the proposed structure. A prototype of the proposed design was fabricated and results were measured. The measured results show a close agreement with the simulated ones. The proposed design is suitable for DCS $1800 \mathrm{MHz}$, PCS $1900 \mathrm{MHZ}$, UMTS, LTE 2500-2700 MHz, and Wimax 3.5 GHz.
\end{abstract}

\section{Introduction}

Wireless devices nowadays support multiple application bands including GSM850/900, PCS, DCS, and WCDMA [1]. Moreover, the recently proposed Long Term Evolution (LTE) offers high data rates, greater system capacity, coverage, and improved efficiency by incorporating high performance antennas [2]. Fortunately, simultaneous operation is not required in all service bands. This implies that an antenna is supposed to cover a subset of bands at any given instant and subsequently to cover a different subset. Therefore, the frequency reconfigurable antennas have wide applicability and are able to provide multiband coverage by altering their resonance frequency and/or the impedance bandwidth $[3,4]$.

Dielectric resonator antennas have recently attained much attention owing to multiple characteristics that include wide impedance bandwidth, low cost, high radiation efficiency, and ease of fabrication. Their design process is flexible offering a control over numerous design parameters like shape, size, aspect ratio, and permittivity.

Antenna miniaturization is becoming a fundamental requirement for wireless applications. Small, efficient, and multiband antennas must be developed which can satisfy the multiple application requirements and can easily be integrated into mobile devices. The absence of miniaturization techniques, particularly at low frequencies, makes the integration impractical for portable devices. Antenna miniaturization techniques for DRAs include C-shaped slotted ground plane [5], use of copper sheet on DRA face [6], metalized upper and lower part of rectangular DRA [7], and stacking of dielectric resonators [8]. All these techniques though successfully reduce the antenna size, the 3D DRA geometry yet poses severe constraints for integration into compact hand-held devices.

Hybrid antenna structures (combining more than one radiating structure) present a fascinating solution for size reduction as well as a multiband operation. A combination 


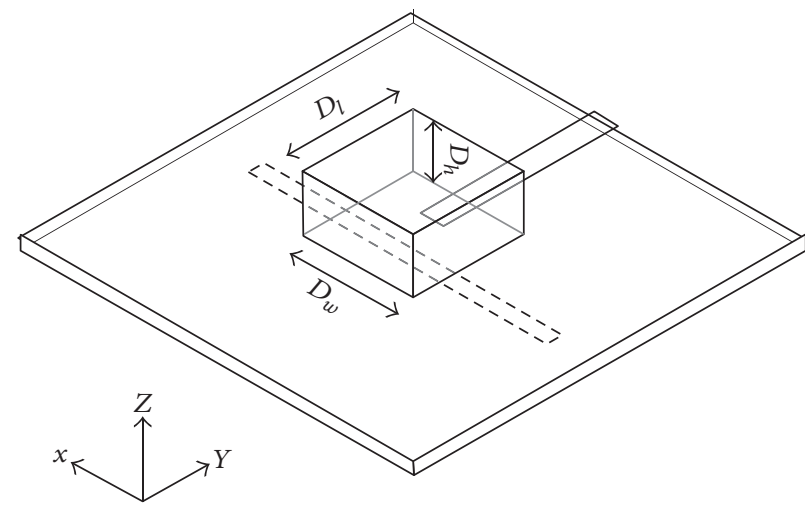

(a)

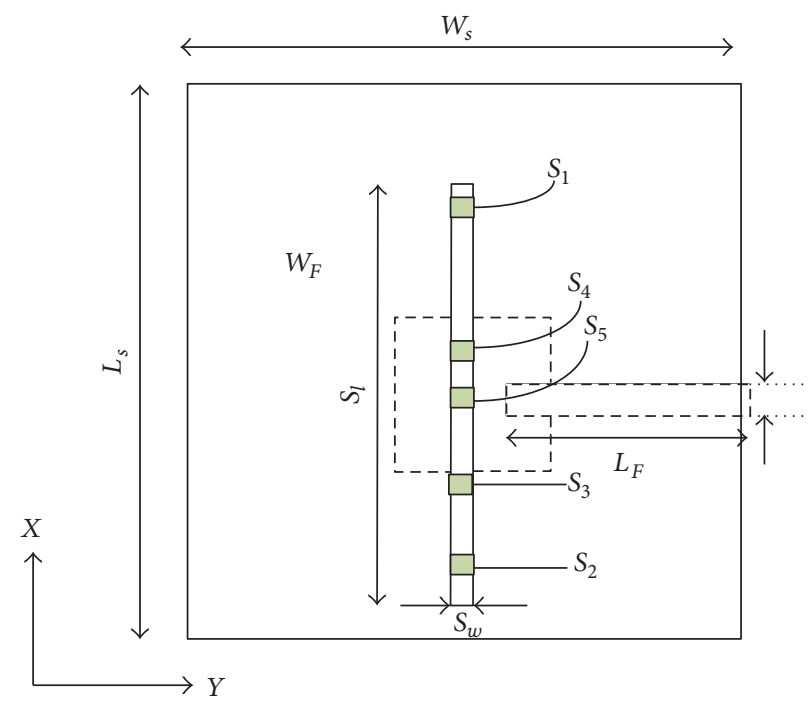

(b)

FIgURE 1: Schematic diagram of the proposed antenna. (a) Front view and (b) back view.

of two ring-shaped slots and a conical shape DRA [9], a combination of T-shaped microstrip line and a circular DRA [10], CPW fed dual band DRA [11], coaxial probe fed RDRA [12], and a combination of moon-shaped defected ground structure with a circular DRA [13] are a few examples of hybrid structures.

In this article, a novel miniaturized reconfigurable hybrid structure consisting of a rectangular DR element coupled with a GP slot is presented. The GP slot acts as a radiator and also assists in shifting the fundamental mode of the DRA by introducing slow wave effect. The combination (of DR and slot) significantly reduces the antenna size and tunes the structure for a multiband operation. As the miniaturization/reconfiguration is achieved through GP slot, there is little effect on the cross-polarization levels.

Section 2 describes the antenna configuration, while the operating principle along with mathematical formulation is presented in Section 3. Section 4 presents a comprehensive analysis of the proposed structure along with parametric details. The tuning capabilities of the proposed antenna are detailed in Section 5, while Section 6 is dedicated to providing the measurement results. Finally, Section 7 concludes the current communication.

\section{Antenna Design and Configuration}

The schematic diagram of the proposed antenna structure is shown in Figure 1. A small rectangular DR element with relative permittivity $\varepsilon_{\mathrm{dra}}=30$ is glued to a system grounded FR4 substrate with a relative permittivity of $\left(\varepsilon_{s}\right) 4.4$ and a loss $\operatorname{tangent}(\tan \delta)$ of 0.02 . Figure $1(\mathrm{~b})$ shows the system ground plane on which a rectangular slot with dimensions $\left(S_{l} \times S_{w}\right)$ is engraved. Five ideal switches $(2 \mathrm{~mm} \times 2 \mathrm{~mm}$ copper tabs for simulation purposes), namely, $S_{1}, S_{2}, S_{3}, S_{4}$, and $S_{5}$, are appropriately located on the slot. The position of switches is optimized through a parametric study. The ON-OFF combinations of switches generate five useful configurations (Config-I through Config-V). The optimized dimensions of the proposed antenna are summarized in Table 1.

\section{Working Principle}

The combination of DR and slot yields a double resonant structure with low cross-polarization levels and good radiation characteristics [15]. Both structures (DR and GP slot) are coupled and a minor change in either structure parameter also affects the other resonance. However, as a first-order approximation, both structures are treated independently.

The size of the dielectric resonator can be approximated by dielectric waveguide model (DWM). According to the dielectric waveguide model, the field components of the $\mathrm{TE}_{111}^{x}$ mode are given as [16]

$$
\begin{aligned}
k_{x} \tan \left(\frac{k_{x} b}{2}\right) & =\sqrt{\left(\varepsilon_{\mathrm{DRA}}-1\right) k_{0}^{2}-k_{x}^{2}} \\
k_{x}^{2}+k_{y}^{2}+k_{z}^{2} & =\varepsilon_{\mathrm{DRA}} k_{0}^{2} \\
k_{y} & =\frac{\pi}{b} \\
k_{z} & =\frac{\pi}{d}
\end{aligned}
$$

where $k_{0}$ is the free space wavenumber, $\varepsilon_{\mathrm{DRA}}$ is the relative permittivity of the DRA, and $k_{x}, k_{y}, k_{z}$ are the wavenumbers in $x, y$, and $z$ directions, respectively. The $E$-field plots in the $Y Z$-plane and $X Z$-plane, shown in Figure 2, confirm the existence of $\mathrm{TE}_{111}^{x}$ mode inside the DR element.

The $S$-parameters of the DRA (in the absence of slot on the GP) are shown in Figure 3. 
TABLE 1: Dimensions of the proposed FR DRA.

\begin{tabular}{|c|c|c|c|}
\hline S. number & Design parameters & Dimensions & Material used \\
\hline (1) & $\begin{array}{l}\text { Substrate length } L_{s} \\
\text { Substrate width } W_{s} \\
\text { Substrate height } H_{s}\end{array}$ & $\begin{array}{c}L_{s} \times W_{s} \times H_{s} \\
50 \times 50 \times 1.6 \mathrm{~mm}^{3}\end{array}$ & $\begin{array}{l}\mathrm{FR} 4\left(\varepsilon_{s}=4.4\right. \\
\tan \delta=0.002)\end{array}$ \\
\hline (2) & $\begin{array}{l}\text { Ground plane length } G_{l} \\
\text { Ground plane width } G_{w} \\
\text { Ground plane thickness } G_{h}\end{array}$ & $\begin{array}{c}L_{g} \times W_{g} \times H_{g} \\
50 \times 50 \times 0.035 \mathrm{~mm}^{3}\end{array}$ & Copper \\
\hline (3) & $\begin{array}{l}\text { DRA length } D_{l} \\
\text { DRA width } D_{w} \\
\text { DRA height } D_{h}\end{array}$ & $\begin{array}{c}D_{l} \times D_{w} \times D_{h} \\
14 \times 14 \times 6 \mathrm{~mm}^{3}\end{array}$ & $\begin{array}{c}\text { Alumina } \\
\left(\varepsilon_{\mathrm{DRA}}=30\right) \\
\tan \delta=0.002\end{array}$ \\
\hline (4) & $\begin{array}{l}\text { Width of microstrip line } W_{F} \\
\text { Length of microstrip line } L_{F}\end{array}$ & $\begin{array}{l}W_{F}=3 \mathrm{~mm} \\
L_{F}=22 \mathrm{~mm}\end{array}$ & Copper \\
\hline (5) & $\begin{array}{l}\text { Length of slot } S_{l} \\
\text { Width of slot } S_{w}\end{array}$ & $\begin{array}{l}S_{l}=36 \mathrm{~mm} \\
S_{w}=2 \mathrm{~mm}\end{array}$ & \\
\hline
\end{tabular}

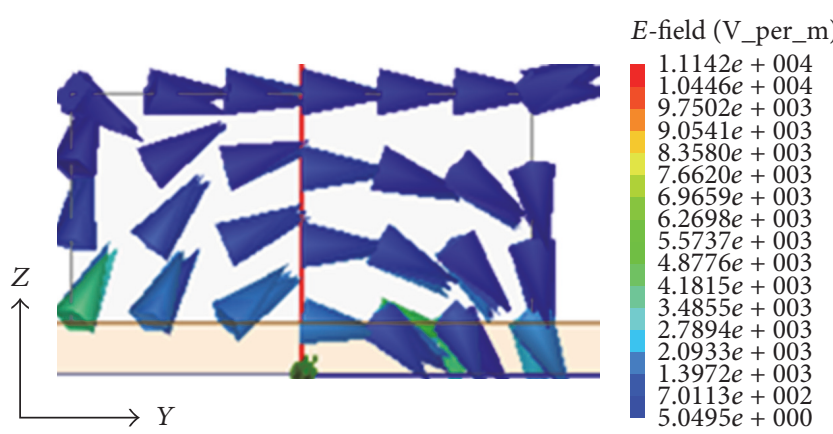

(a)

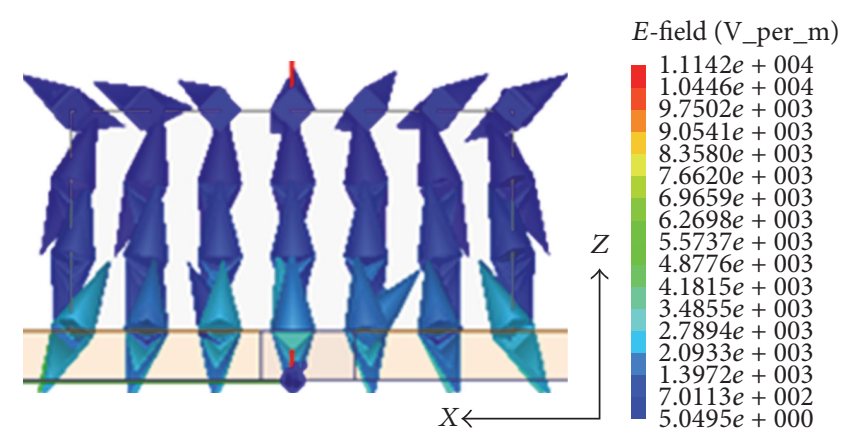

(b)

FIgURE 2: E-field distribution on DRA. (a) YZ-plane and (b) XZ-plane.

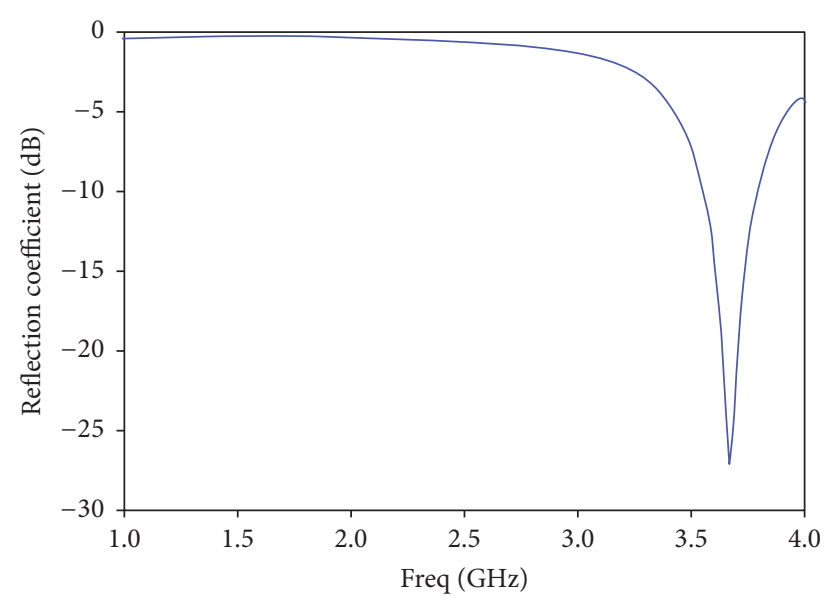

FIGURE 3: S-parameters of the DRA, without a slot on the ground plane.

The slot resonance frequency (without DR element) can be approximated as [17]

$$
f_{0}=\frac{m c}{2 L_{s} \sqrt{\epsilon_{\mathrm{eff}}}}
$$

where

$$
\epsilon_{\mathrm{eff}}=\frac{1}{2}\left(\varepsilon_{s}+1\right)+\frac{1}{2}\left(\varepsilon_{s}-1\right)\left(1+\frac{10 H_{s}}{W}\right),
$$

where $m=1,2,3, \ldots, \epsilon_{\text {eff }}$ is the effective permittivity of substrate, $c$ is the speed of light, $H_{s}$ is the height of substrate, and $W$ is the width of slot. From the above equations, the resonance frequency of the slot is found to be $2.3 \mathrm{GHz}$ for $m=1$. The reflection coefficient for the slot (without DR element) is shown in Figure 4. Although the matching is poor, it, however, verifies the resonant behavior of the slot.

The placement of DR above the slotted ground plane introduces an additional relative permittivity for the slot. The additional relative permittivity due to presence of DR element is given as [15]

$$
\epsilon_{\text {add }}=\frac{H_{\text {total }}}{D_{h} / \varepsilon_{r, \text { upper }}+H_{s} / \varepsilon_{s}},
$$

where $H_{\text {total }}$ is the total height of the antenna structure, $D_{h}$ is the height of DR element, $H_{s}$ is the height of substrate with relative permittivity $\varepsilon_{s}$, and

$$
\varepsilon_{r, \text { upper }}=\frac{\varepsilon_{\mathrm{DRA}} \times \varepsilon_{\mathrm{air}}}{\varepsilon_{\mathrm{DRA}}+\varepsilon_{\mathrm{air}}},
$$




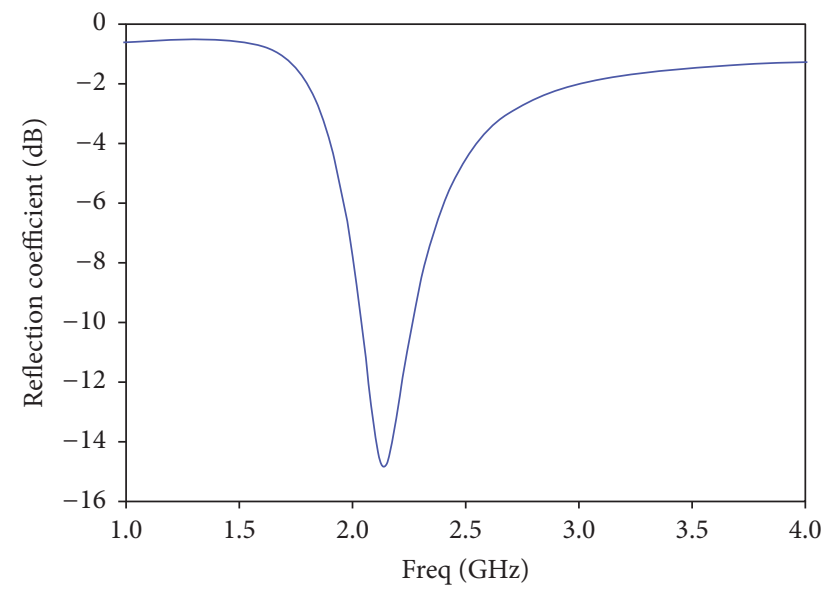

FIGURE 4: Slot resonance frequency without DRA.

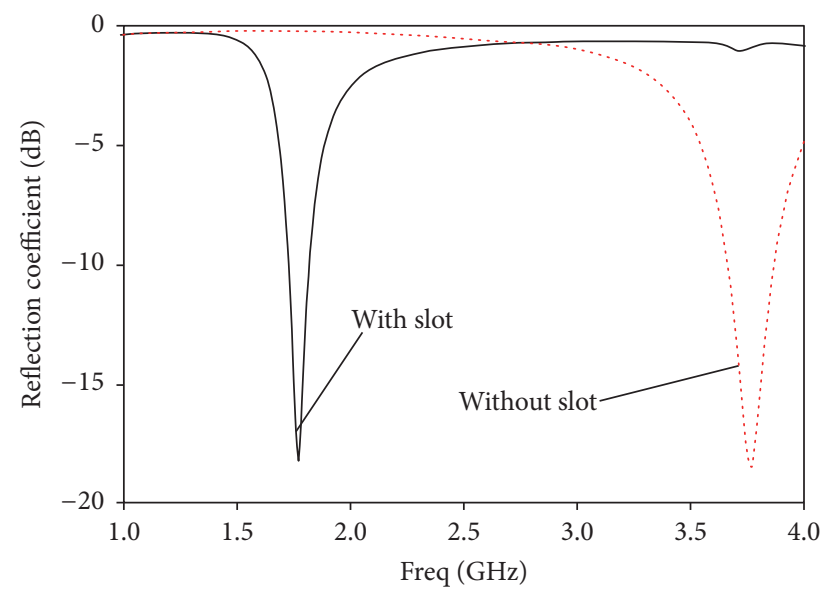

FIGURE 5: Variation in resonance frequency with and without slot.

where $\varepsilon_{\mathrm{DRA}}$ and $\varepsilon_{\text {air }}$ are the relative permittivities of DR element and air, respectively.

The additional relative permittivity due to the DR presence further shifts the slot resonance downwards. The calculated slot resonance frequency (with DR) is found to be $1.83 \mathrm{GHz}$. The simulated resonance frequency is $1.77 \mathrm{GHz}$ as shown in Figure 5.

A volumetric source (like DR) improves the radiation power factor (RPF) of the slot, besides lowering its resonance frequency. The RPF was defined by Wheeler [18], in terms of the volume of the antenna:

$$
\mathrm{RPF} \propto \frac{V_{a}}{V_{s}},
$$

where $V_{a}$ represents the effective antenna volume and $V_{s}$ is the volume of radian sphere given as $V_{s}=(4 \pi / 3)(\lambda / 2 \pi)^{3}$. As $V_{a}$ is much larger in case of DRA, the RPF of the slot is greatly improved.

\section{Antenna Analysis}

The proposed antenna optimization and parametric analysis is performed using Ansoft HFSS. By properly combining the slot and DRA resonances, the antenna size can be miniaturized significantly. Figure 5 shows the reflection coefficient of the antenna with and without GP slot. It is clear from Figure 5 that the presence of GP slot reduces the structure resonance frequency by more than $50 \%$ (from $3.68 \mathrm{GHz}$ to $1.77 \mathrm{GHz}$ ). Both resonant structures (DRA and slot) are responsible for resonating the structure at such a lower frequency. The DRA presence alters the effective permittivity for the slot, while slot presence introduces a slow wave effect which causes the DRA to resonate at a much lower frequency. To validate these facts, the resonance frequency, 3D gain plot, $E$-field distribution on DRA, and the surface current distributions on the ground plane of the antenna are shown in Figure 6. The surface current distribution shown in Figure 6(d) verifies the resonant behavior of the slot which is further confirmed by observing the 3D gain plot in Figure 6(b). The DRA, however, resonates in its fundamental mode (i.e., $\mathrm{TE}_{111}^{x}$ ) which is evident from Figure 6(c).

Antenna compactness has a paramount importance for 3D structures like DRA. It enables them to be integrated in small portable devices. A comparison of the proposed design with existing DRA based design in terms of compactness is provided in Table 2. The comparison reveals that the proposed design technique outperforms the published work in terms of compactness.

4.1. Parametric Analysis. A parametric analysis was performed to study the effects of slot length, width, and slot positions. Figure 7 shows the variation of reflection coefficient for different values of slot length $S_{l}$. The slot length $\left(S_{l}=0\right)$ corresponds to a conventional ground plane. Figure 7 shows that the resonance frequency decreases with an increase in the slot length. Equation (2) confirms an inverse relationship between slot length and its resonance frequency.

Another parametric analysis was performed to study the resonance frequency dependence on slot width. Figure 8 shows the reflection coefficient variation for different values of slot width $S_{w}$. It is evident that slot width does not have a profound effect on the reflection coefficient.

Figure 9 shows the resonance frequency variation for different slot positions, that is, a centered position slot and shifted positions in the $+x$-direction and $-x$-direction. In case of the centered position slot, the antenna resonates at $1.77 \mathrm{GHz}$, while, in case of shifted positions (either in the $+x$-direction or $-x$-direction), the DRA resonates at its original frequency (i.e., around $3.6 \mathrm{GHz}$ ). Thus slot position plays a very important role in obtaining the lower resonance frequency. When placed right beneath the DRA (centered position), it acts as a feed structure for the DRA, while the DRA acts as a superstate for the slot.

\section{Frequency Reconfigurability}

The parametric analysis shows the potential of effectively combining the two resonant structures for size miniaturization and frequency reconfiguration. Figure 7 shows that an increase (decrease) in slot length decreases (increases) 


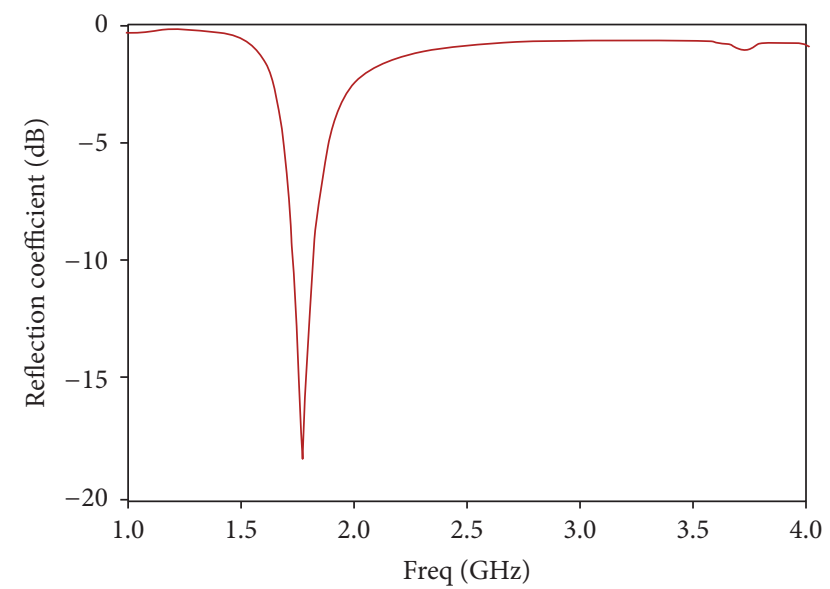

(a)

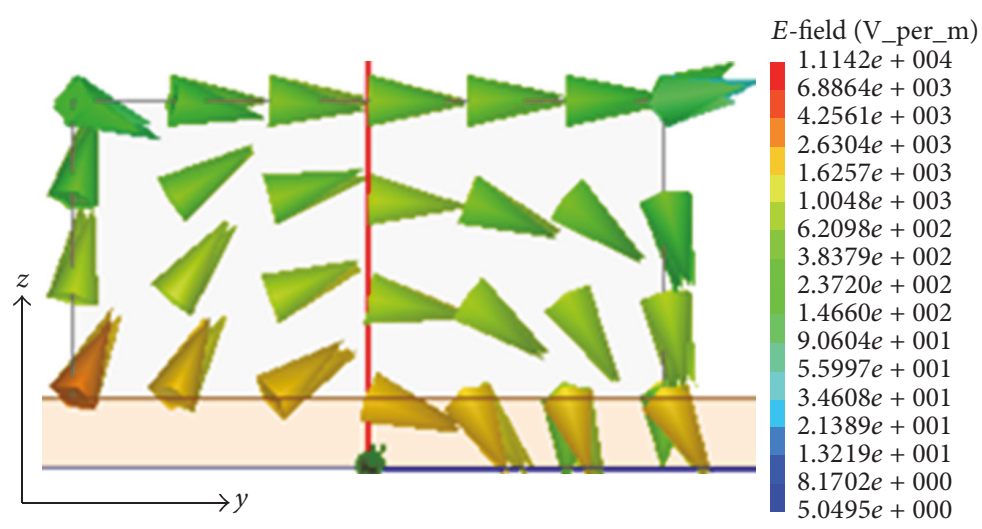

(c)

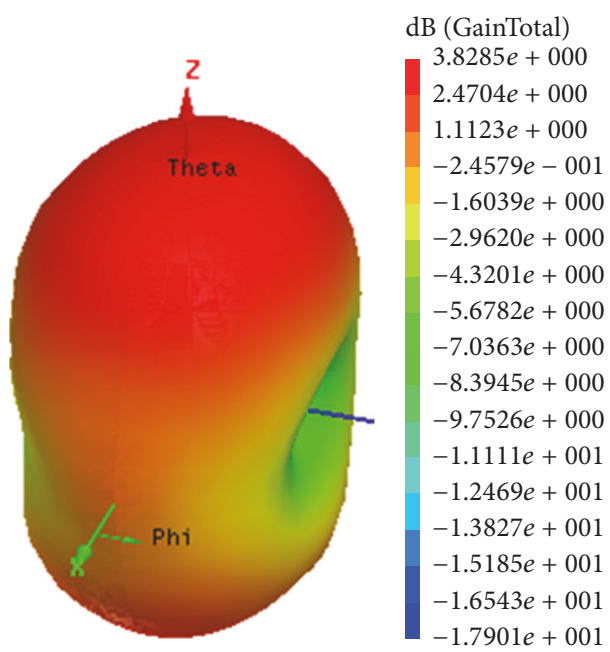

(b)

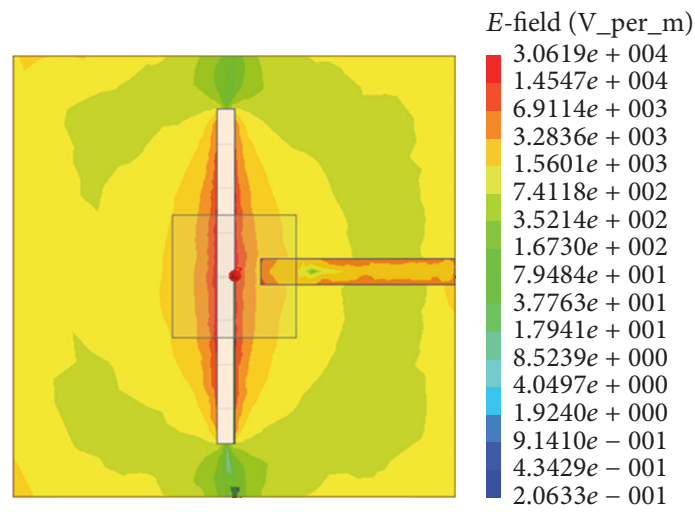

(d)

FiguRE 6: Antenna parameters in presence of slot. (a) Reflection coefficient, (b) 3D gain plot, (c) E-field distribution on DRA at 1.77 GHz, and (d) surface current distribution on ground plane at $1.77 \mathrm{GHz}$.

TABLE 2: Comparison between DRAs with proposed structure on the basis of compactness.

\begin{tabular}{lccc}
\hline S. number & DRA shapes & Technique used & Compactness (\%) \\
\hline$(1)$ & Cylindrical DRA [6] & C-shaped slot & 5 \\
$(2)$ & Rectangular DRA [7] & Metallic patch & 39 \\
$(3)$ & Rectangular DRA [14] & DRA on patch & 23 \\
$(4)$ & Ring DRA [13] & Moon-shaped DGS & 48.77 \\
$(5)$ & This work & Rectangular slot DGS & 53.54 \\
\hline
\end{tabular}

the resonance frequency. The principle can effectively be applied to design a frequency reconfigurable antenna by placing switches $(2 \mathrm{~mm} \times 2 \mathrm{~mm}$ copper tabs $)$ on the GP slot. A switch placement along the GP slot introduces a new current path which reduces the effective slot length and results in an increase of resonance frequency. Figure 12 shows surface current distributions for five different configurations. The switch placement alters the surface current on the ground plane, thereby, changing the resonance frequency. The resonance frequency shift depends upon the location of switch (switches). Five useful configurations, summarized in Table 3, arise from the ON/OFF combinations of switches. In
Config-I (all switches in OFF state), the structure resonates with a center frequency of $1.77 \mathrm{GHz}$, while, in Config-V $\left(S_{3}, S_{4}, S_{5} \mathrm{ON} ; S_{1}, S_{2} \mathrm{OFF}\right)$, the resonance frequency is shifted to $3.62 \mathrm{GHz}$. In other configurations, the resonance frequency remains between these two extremes.

Both DR and GP slots resonate simultaneously, with DR element acting as a superstate for the slot which brings down the slot resonance as discussed earlier. The original resonance of the DR element is also shifted from $3.68 \mathrm{GHz}$ to $1.77 \mathrm{GHz}$ in presence of slot. The slot acts as a defected ground structure (DGS) for the DRA which introduces a slow wave effect. The slow wave effect enhances the effective permittivity of the 
TABLE 3: Various configurations of the proposed FR antenna.

\begin{tabular}{lcccc}
\hline S. number & Config-name & Switches ON state & Switches OFF state & Resonance frequency (GHz) \\
\hline$(1)$ & Config-I & & $S 1, S 2, S 3, S 4, S 5$ & 1.77 \\
$(2)$ & Config-II & $S 1$ & $S 2, S 3, S 4, S 5$ & 1.9 \\
$(3)$ & Config-III & $S 2$ & $S 1, S 3, S 4, S 5$ & 2.03 \\
$(4)$ & Config-IV & $S 3$ & $S 1, S 2, S 4, S 5$ & 2.6 \\
$(5)$ & Config-V & $S 3, S 4, S 5$ & $S 1, S 2$ & 3.68 \\
\hline
\end{tabular}

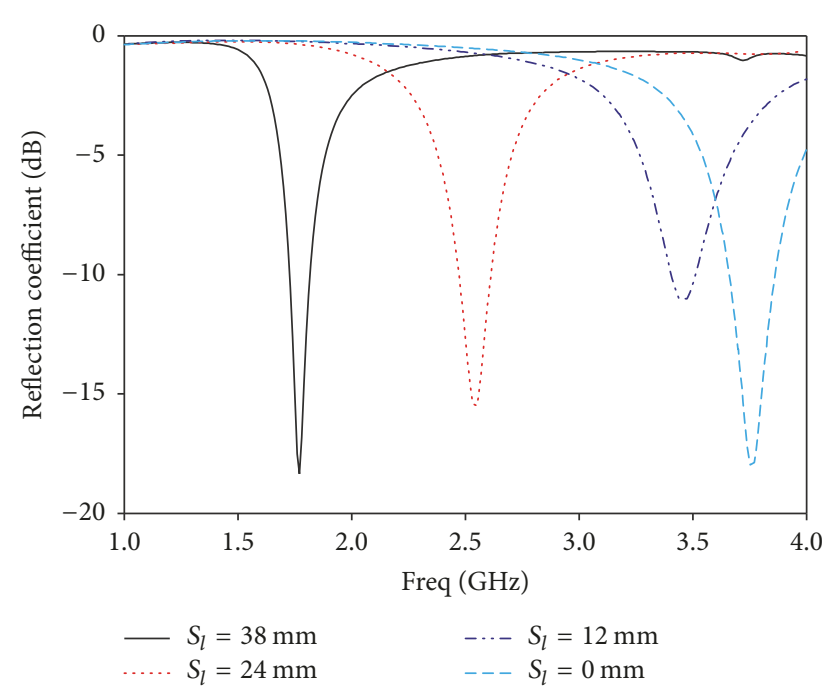

FIGURE 7: Resonance frequency variation for different slot lengths.

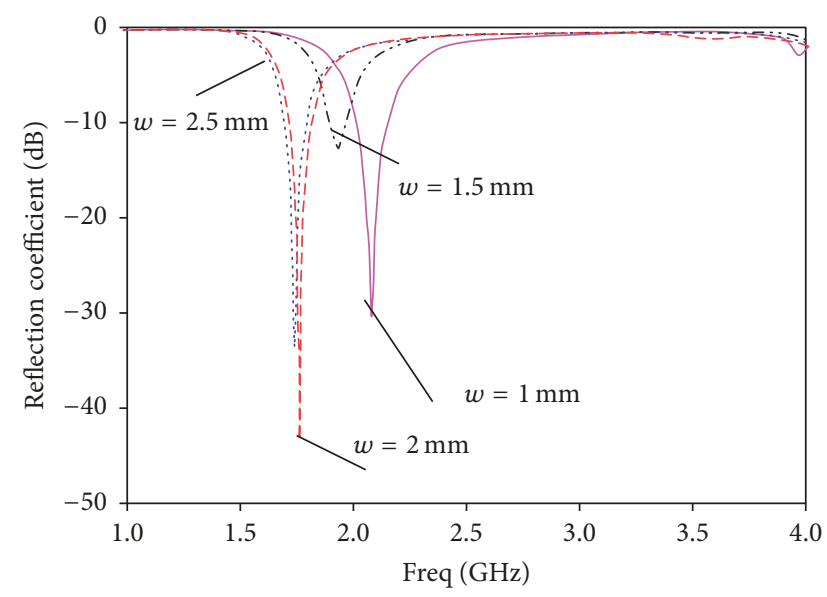

FIGURE 8: Resonance frequency variation for different slot widths.

DRA. The resonance frequency of the DRA is decreased with an increase in the effective permittivity. A similar reduction in DRA resonance has also been reported in [13]; however, the work in [13] treats the slot resonance and DRA resonance independently, while, in our work, the two resonances act in unison. The placement of switches on the slot alters the effective DGS slot length and hence the resonance frequency is shifted upwards. To analyze the slow wave effects of the DGS slot, the $S_{21}$ parameters of different configurations are simulated and are shown in Figure 10. Slow wave effects occur

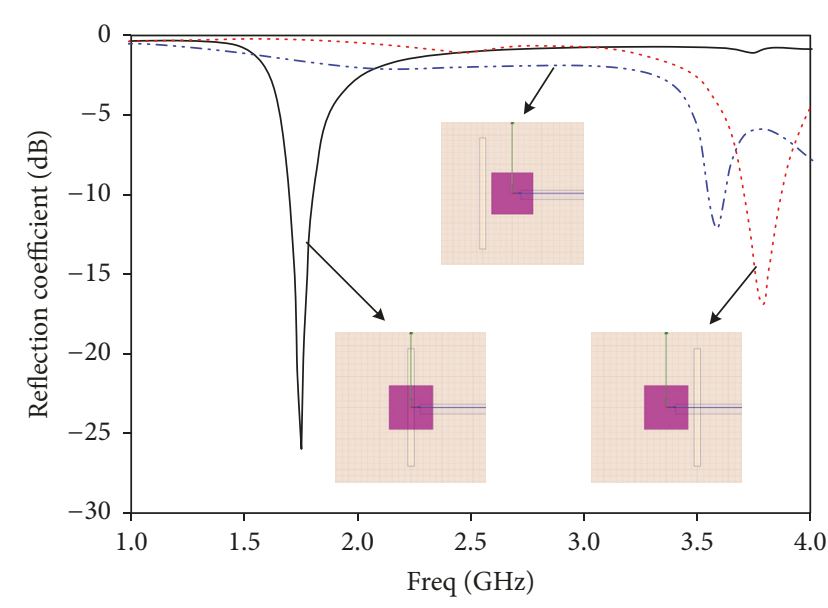

FIGURE 9: Resonance frequency variation for different slot positions.

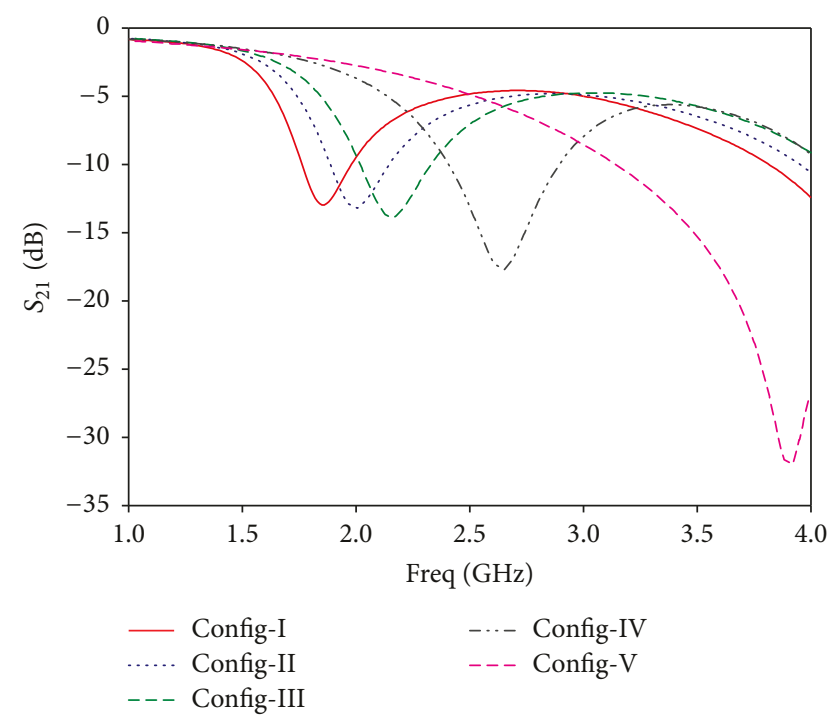

FIGURE 10: Transfer characteristics of the GP slot for five different configurations. (a) Config-I, (b) Config-II, (c) Config-III, (d) Config-IV, and (e) Config-V.

when the operating frequency is less than the stop band frequency of DGS. From Figure 10, it is clear that the SBF of DGS shifts in each configuration. In all configurations, the DRA resonates in its fundamental mode which can be verified by observing the $E$-field distributions shown in Figure 11. The slot, however, is also resonant in the first three configurations (Config-I, Config-II, and Config-III) which can be verified 


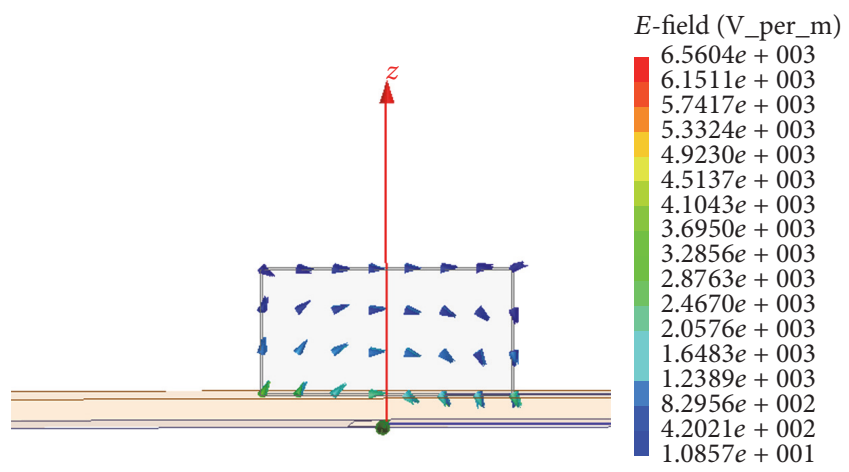

(a)

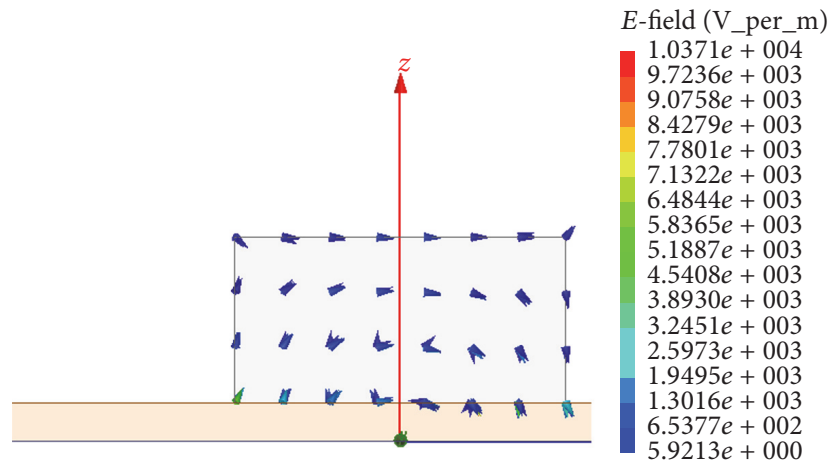

(c)

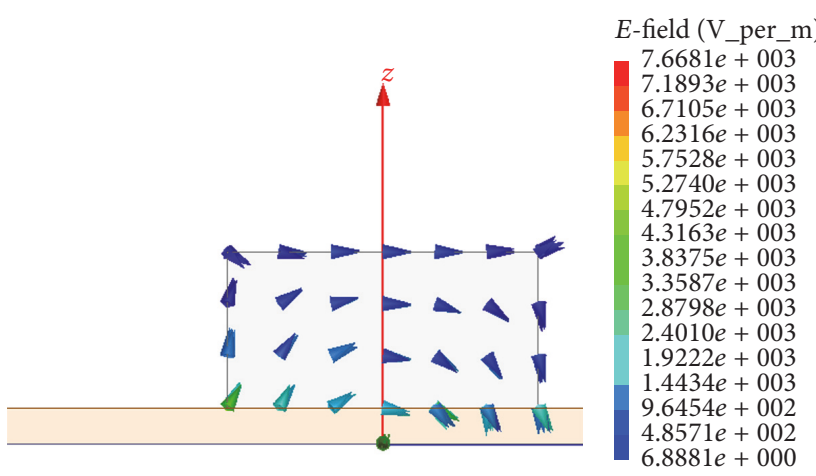

(b)

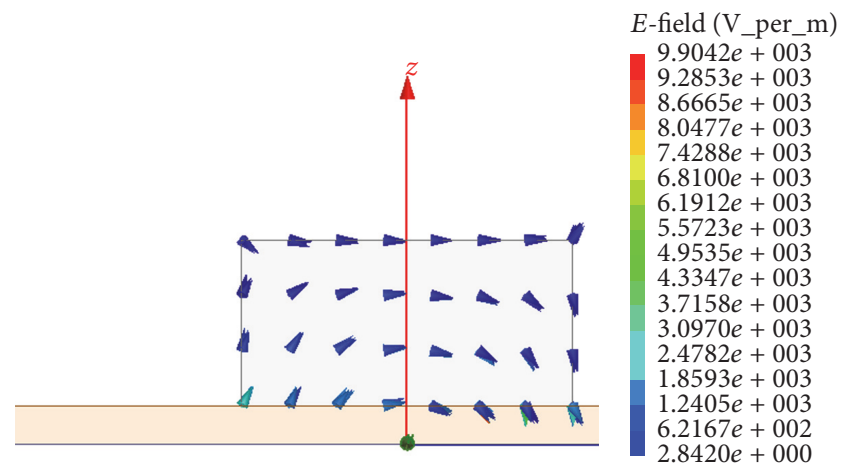

(d)

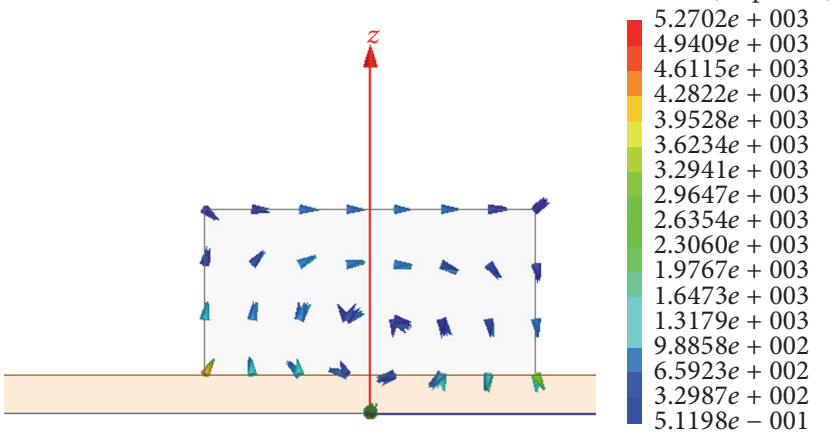

(e)

Figure 11: E-field distributions on rectangular DRA. (a) Config-I, (b) Config-II, (c) Config-III, (d) Config-IV, and (e) Config-V.

by the surface current distributions shown in Figure 12. The behavior can be further verified by observing the 3D gain plots of the five configurations shown in Figure 13. The slot behaves like a magnetic dipole which is evident from Figures 13(a), 13(b), and 13(c), corresponding to Config-I, Config-II, and Config-III, respectively. Figures 13(d) and 13(e) show the $3 \mathrm{D}$ gain plot for Config-IV and Config-V. The DRA is the only radiating source for these two configurations which is evident from the gain plots.

The simulated reflection coefficients corresponding to the five configurations are shown in Figure 14.

\section{Experimental Results}

The proposed frequency reconfigurable design was fabricated to corroborate the simulated results. A photograph of antenna is shown in Figure 15. The ideal conducting tabs were replaced by PIN diodes BAR-64-02 V. The PIN diodes were biased by $\mathrm{DC}$ bias lines which are terminated by inductors $(68 \mathrm{nH})$. The inductors prevent the leakage of RF signal to the biasing circuitry. Figure 15 also shows $0.3 \mathrm{~mm}$ DC slot lines for the purpose of ground isolation. A number of $100 \mathrm{pF}$ capacitors were placed on the DC slot lines for DC blocking and to provide effective RF wave connection. 


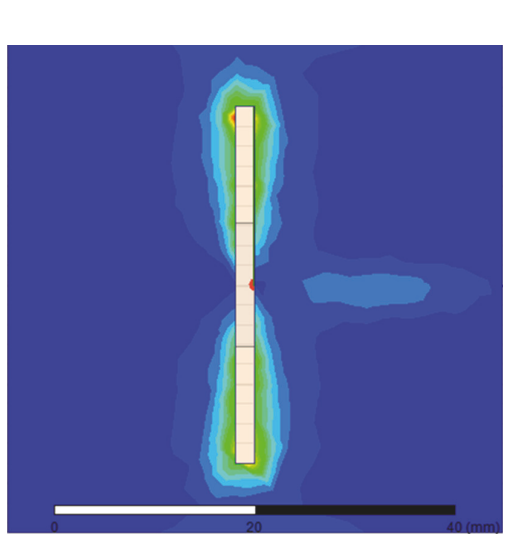

(a)

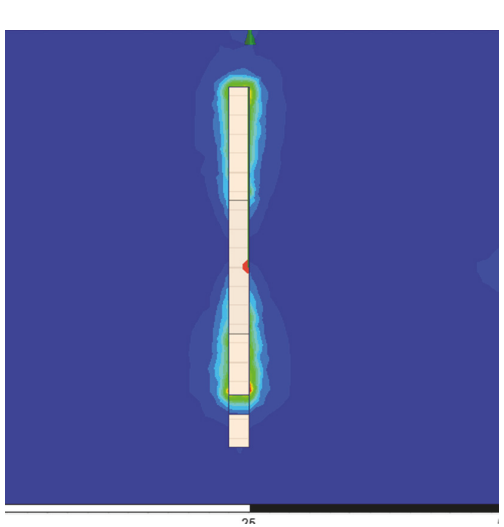

(c)
Jsurf (A per_m)

$2.0046 e+002$

$1.8794 e+002$

$1.7541 e+002$

$1.6288 e+002$

$1.5036 e+002$

$1.3783 e+002$

$1.2530 e+002$

$1.1277 e+002$

$1.0025 e+002$

$8.7721 e+001$

$7.5195 e+001$

$6.2668 e+001$

$5.0141 e+001$

$3.7614 e+001$

$2.5088 e+001$

$1.2561 e+001$

$3.4167 e-002$

Isurf (A_per_m) $2.7380 e+002$ $2.5669 e+002$

$2.3958 e+002$

$2.2247 e+002$

$2.0535 e+002$

$1.8824 e+002$

$1.7113 e+002$

$1.5402 e+002$

$1.3691 e+002$

$1.1980 e+002$

$1.0269 e+002$

$8.5575 e+001$

$6.8464 e+001$

$5.1353 e+001$

$3.4242 e+001$

$1.7130 e+001$

$1.9082 e-002$

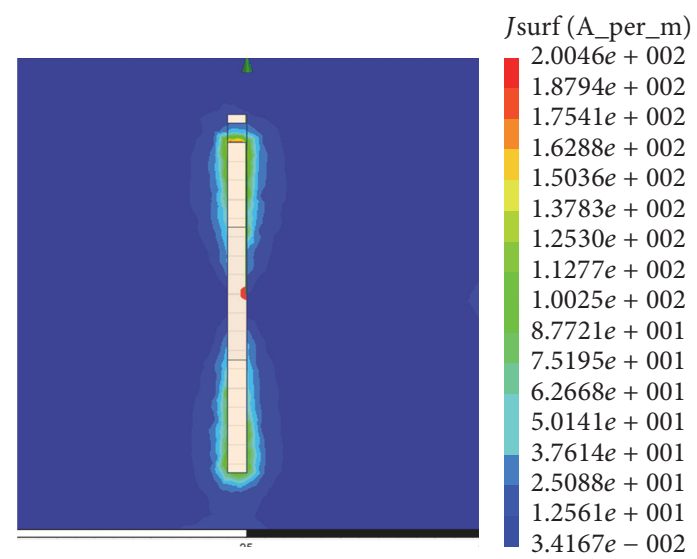

(b)

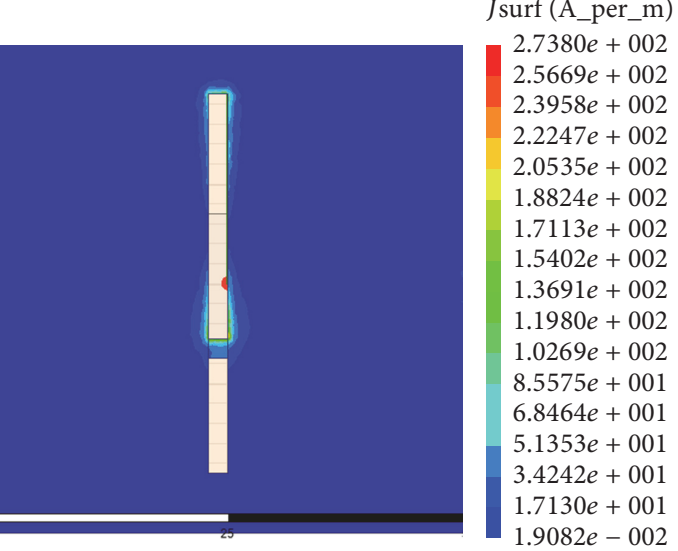

(d)

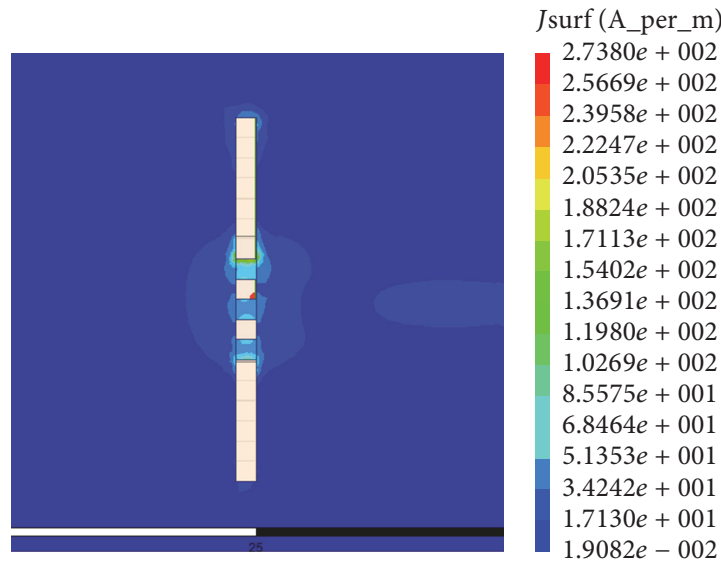

(e)

FIGURE 12: Surface current distribution on the ground plane for five configurations. (a) Config-I, (b) Config-II, (c) Config-III, (d) Config-IV, and (e) Config-V.

The reflection coefficients of the proposed antenna were measured by Agilent E $5071 \mathrm{C}$ network analyzer. The simulated and measured reflection coefficients corresponding to five configurations are shown in Figure 16.

The comparison reveals a close agreement between the simulated and measured results. The slight difference (in a few configurations) may be attributed to fabrication accuracy, diode package parasitic, and/or the improper DC bias network.

Simulated and measured co- and cross-polarization $E$ plane and $H$-plane radiation patterns corresponding to five configurations are shown in Figure 17. The gain patterns are broadside in all configurations with a good isolation between co- and cross-polarization levels. The cross-polarization is 


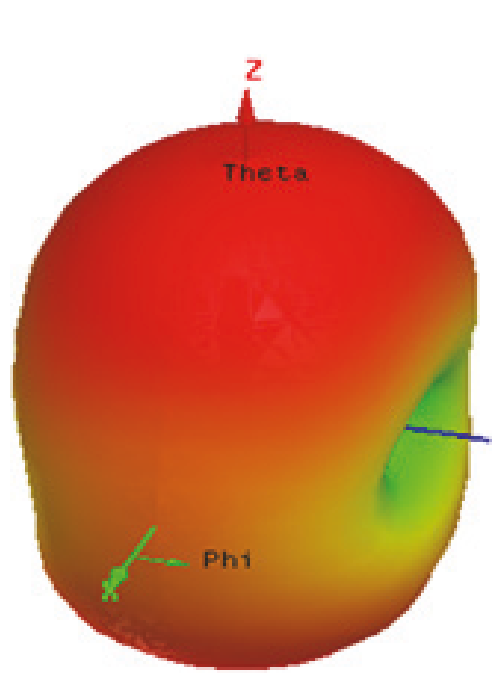

(a)

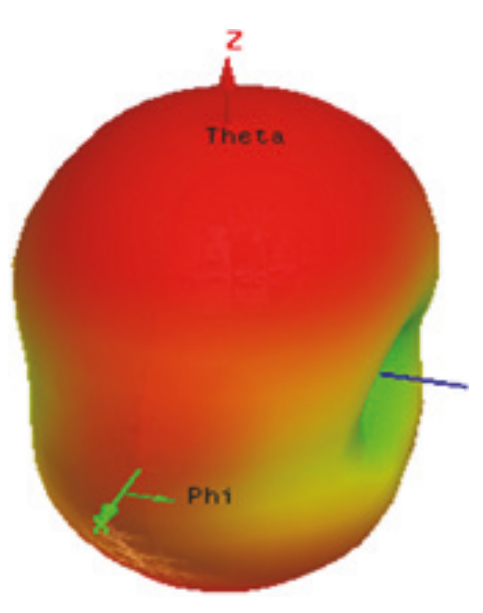

(c)
dB (GainTotal)

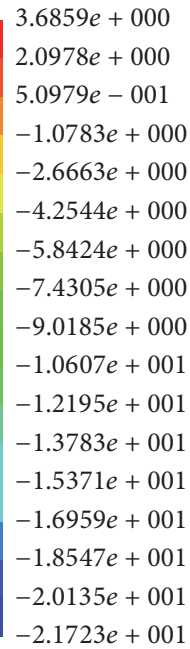

$-2.1723 e+001$

dB (GainTotal)

$3.6608 e+000$

$2.2679 e+000$

$8.7513 e-001$

$-5.1768 e-001$

$-1.9105 e+000$

$-3.3033 e+000$

$-4.6961 e+000$

$-6.0889 e+000$

$-7.4817 e+000$

$-8.8745 e+000$

$-1.0267 e+001$

$-1.1660 e+001$

$-1.3053 e+001$

$-1.4446 e+001$

$-1.5839 e+001$

$-1.7231 e+001$

$-1.8624 e+001$

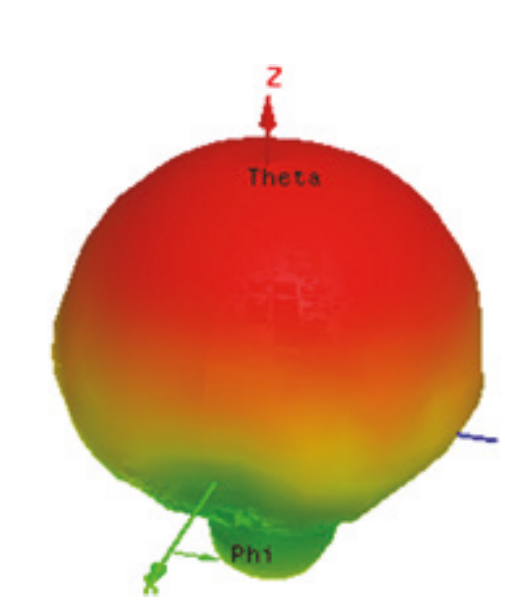

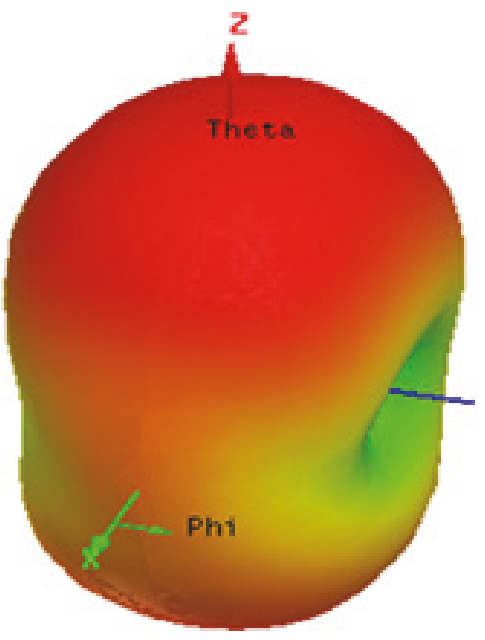

(b)

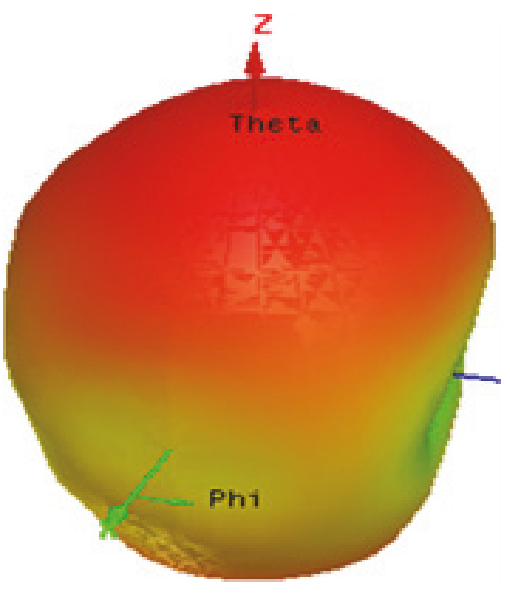

(d)
$\mathrm{dB}$ (GainTotal)

$3.7486 e+000$

$2.3616 e+000$

$9.7459 e-001$

$-4.1242 e-001$

$-1.7994 e+000$

$-3.1864 e+000$

$-4.5734 e+000$

$-5.9604 e+000$

$-7.3474 e+000$

$-8.7344 e+000$

$-1.0121 e+001$

$-1.1508 e+001$

$-1.2895 e+001$

$-1.4282 e+001$

$-1.5669 e+001$

$-1.7056 e+001$

$-1.8443 e+001$

dB (GainTotal)

$5.0208 e+000$

$3.3896 e+000$

$1.7583 e+000$

$1.2704 e-001$

$-1.5042 e+000$

$-3.1355 e+000$

$-4.7668 e+000$

$-6.3980 e+000$

$-8.0293 e+000$

$-9.6606 e+000$

$-1.1292 e+001$

$-1.2923 e+001$

$-1.4554 e+001$

$-1.6186 e+001$

$-1.7817 e+001$

$-1.9448 e+001$

$-2.1079 e+001$

dB (GainTotal)

$6.0520 e+000$

$4.4597 e+000$

$2.8674 e+000$

$1.2750 e+000$

$-3.1729 e-001$

$-1.9096 e+000$

$-3.5020 e+000$

$-5.0943 e+000$

$-6.6866 e+000$

$-8.2789 e+000$

$-9.8713 e+000$

$-1.1464 e+001$

$-1.3056 e+001$

$-1.4648 e+001$

$-1.6241 e+001$

$-1.7833 e+001$

$-1.9425 e+001$

(e)

FIGURE 13: 3D gain plot for the five configurations. (a) Config-I, (b) Config-II, (c) Config-III, (d) Config-IV, and (e) Config-V. 


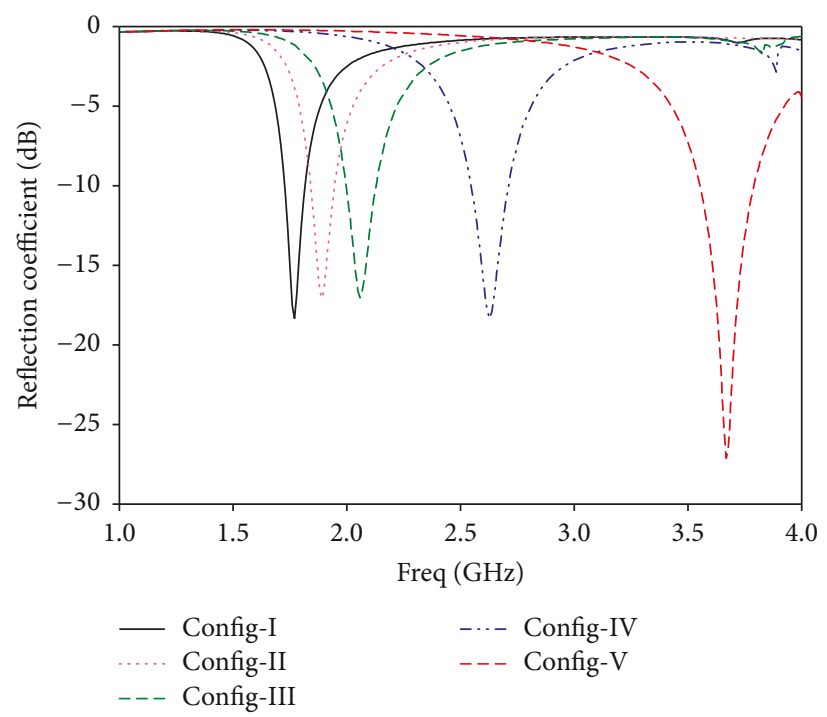

FIGURE 14: Simulated S-parameters corresponding to five different configurations.

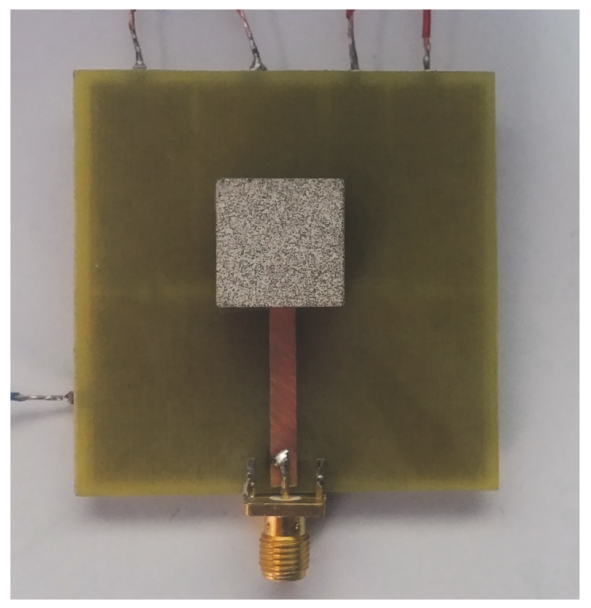

(a)

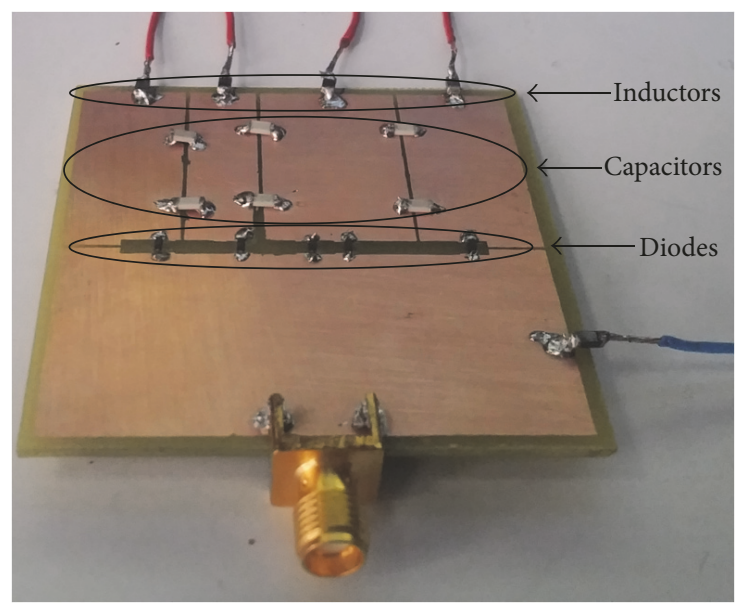

(b)

FIgURE 15: Fabricated proposed antenna. (a) Front view and (b) back view.

well below (more than $15 \mathrm{~dB}$ ) the copolarization in each configuration.

The simulated and measured gain and radiation efficiency for the five configurations are summarized in Table 4. The peak gain values of the proposed antenna tend to increase with an increase in resonance frequency. It is due to the fact that, on lower resonances (Config-I, Config-II), the slot behavior is more dominant while the DRA behavior becomes dominant in Config-IV and Config-V.

\section{Conclusion}

A novel frequency reconfigurable hybrid structure comprising a DR element and GP slot is proposed and analyzed.The proposed antenna is excited with the help of a microstrip line which generates the fundamental mode (i.e., $\mathrm{TE}_{111}^{y}$ ), in the rectangular DR element. Theoretical guidelines coupled with extensive parametric analysis is provided to show that the combination of two resonant structures results in large amount of miniaturization (i.e., 53.54\%). The tuning capabilities of the proposed structure are demonstrated with the help of PIN diodes. The switching combinations of PIN diodes result in five distinct configurations. Another salient feature of the proposed structure is its ability to obtain good radiation efficiency with high gain in all configurations. The generality of the design procedure allows even larger tuning ranges. The obtained configurations are suitable for DCS 1800, PCS 1900, UMTS, LTE 2300-2500, and Wimax 3.5 GHz applications. 


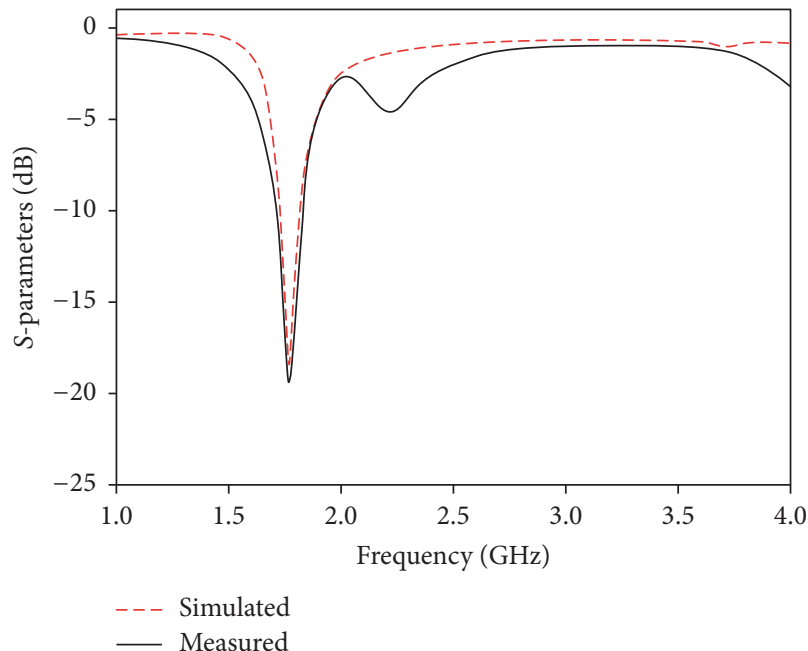

(a)

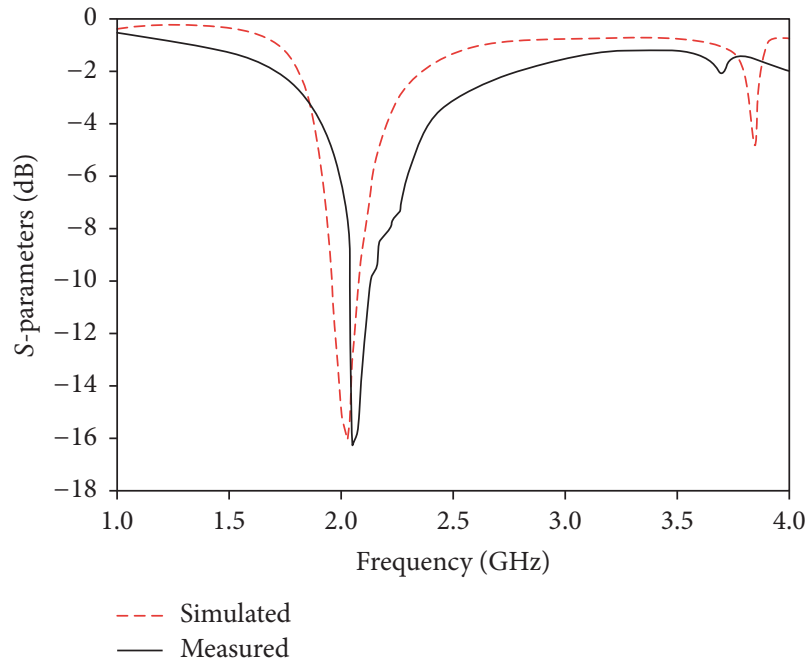

(c)

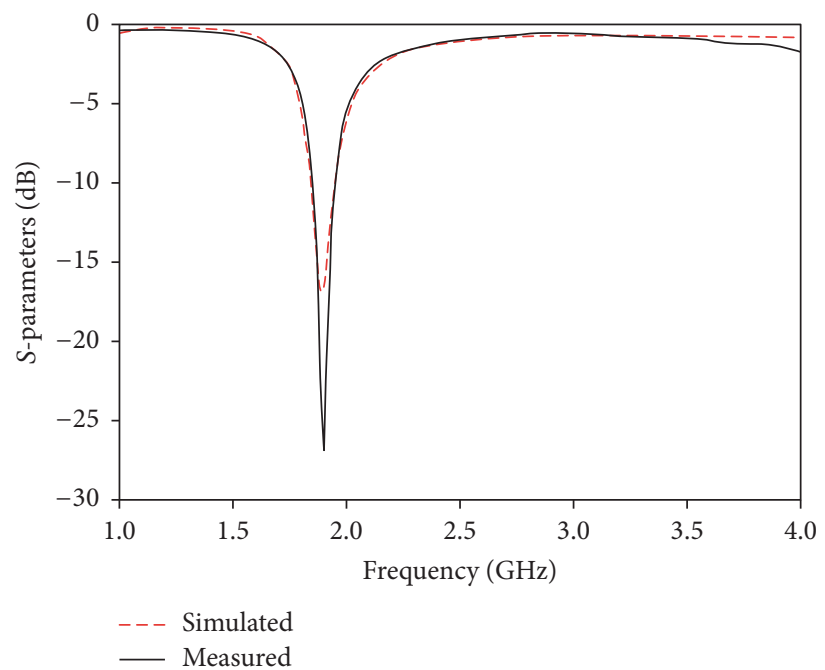

(b)

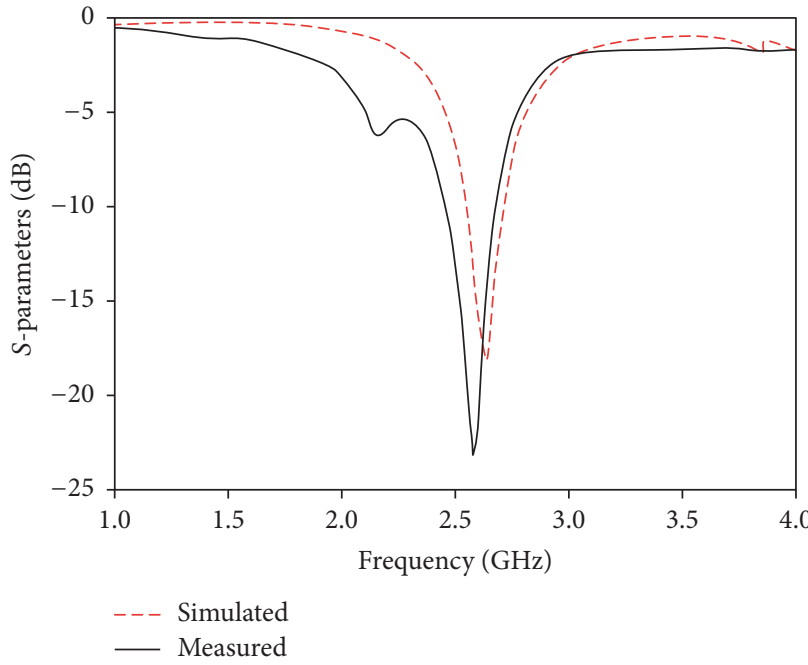

(d)

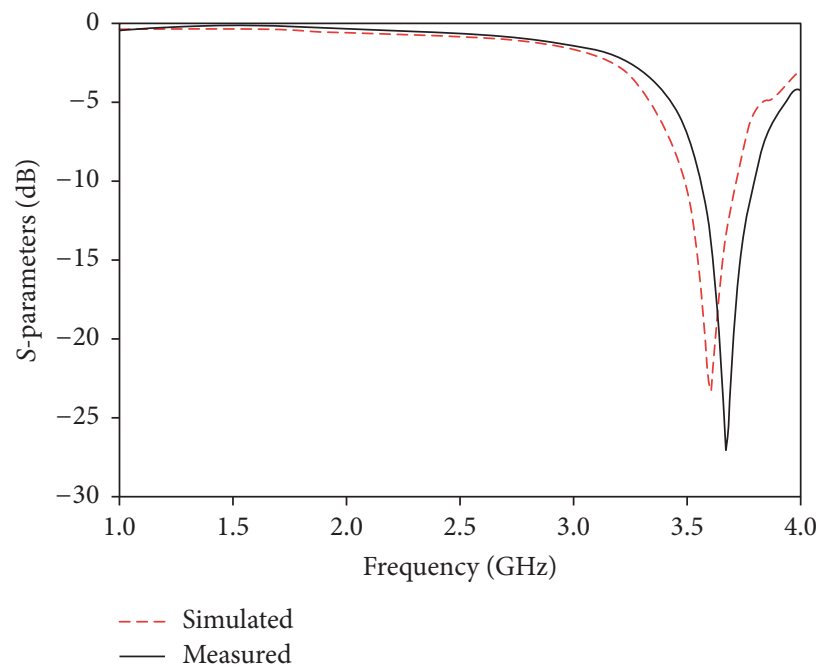

(e)

FIGURE 16: Simulated and measured S-parameters corresponding to five configurations. (a) Config-I, (b) Config-II, (c) Config-III, (d) ConfigIV, and (e) Config-V. 


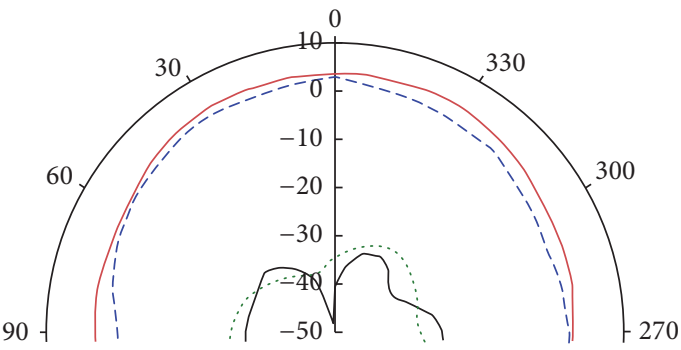

(a)

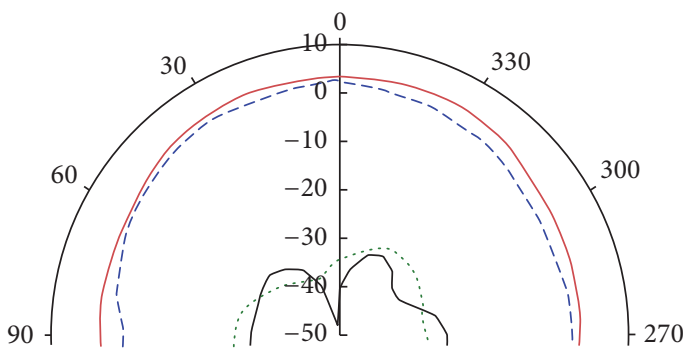

(c)

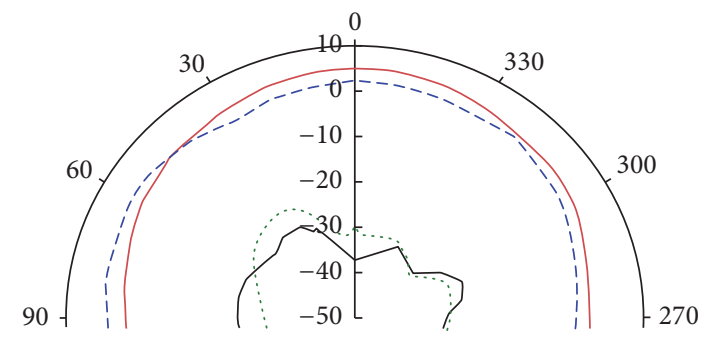

(e)

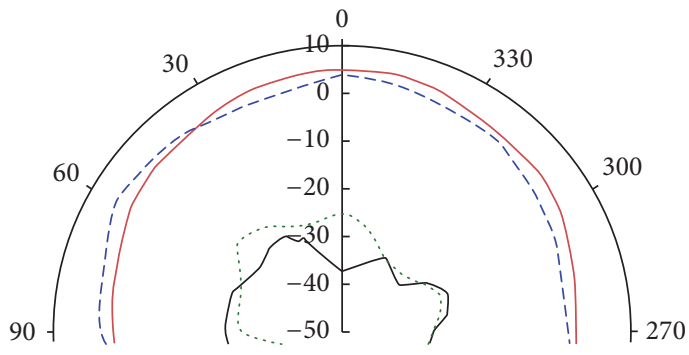

(g)

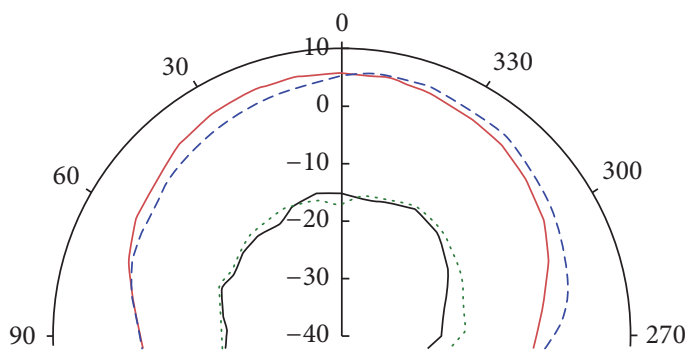

(i)

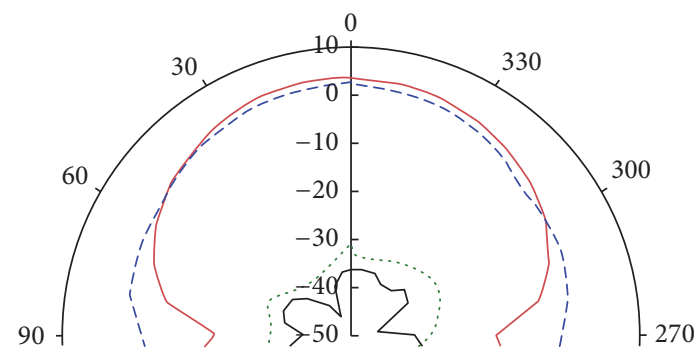

(b)

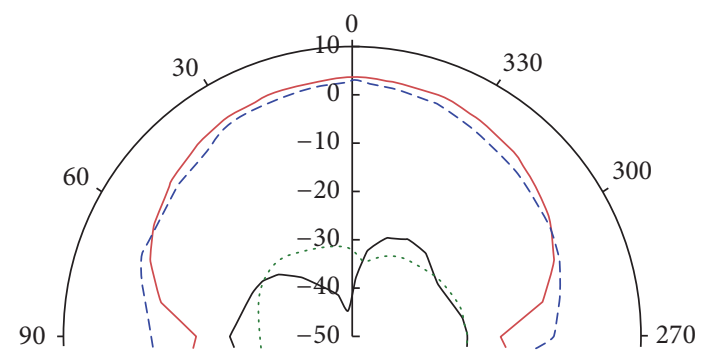

(d)

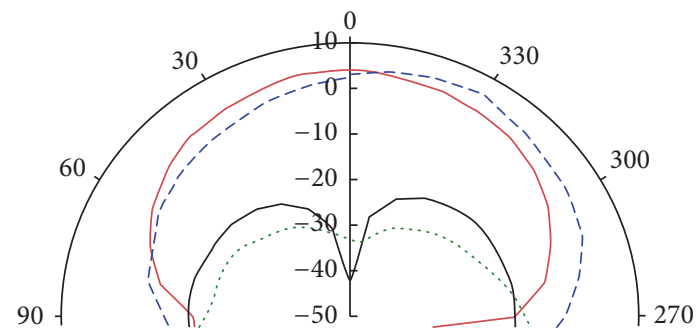

(f)

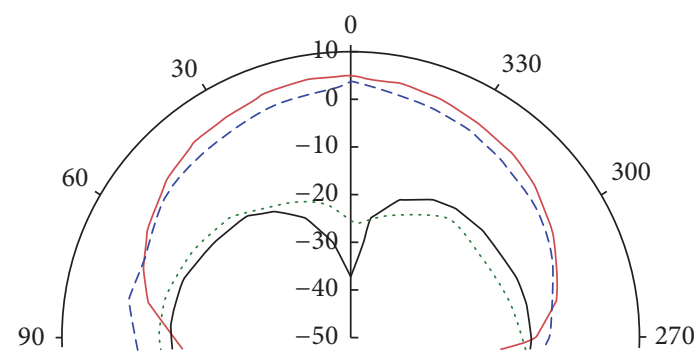

(h)

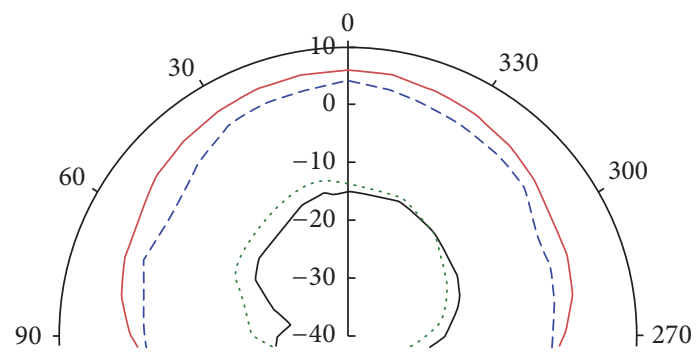

(j)

FigurE 17: Simulated and measured co- and cross-polarization patterns, red solid line: simulated copolarization, blue dotted line: measured copolarization, black solid line: simulated cross-polarization, and green dotted line: measured cross-polarization. (a) E-plane at $1.77 \mathrm{GHz}$, (b) $H$-plane at $1.77 \mathrm{GHz}$, (c) $E$-plane at $1.9 \mathrm{GHz}$, (d) $H$-plane at $1.9 \mathrm{GHz}$, (e) $E$-plane at $2.05 \mathrm{GHz}$, (f) $H$-plane at 2.05 GHz, (g) E-plane at $2.6 \mathrm{GHz}$, (h) $H$-plane at $2.6 \mathrm{GHz}$, (i) $E$-plane at $3.68 \mathrm{GHz}$, and (j) $H$-plane at $2.68 \mathrm{GHz}$. 
TABLE 4: Simulated and measured gain and radiation efficiency of the proposed FR antenna.

\begin{tabular}{lcccc}
\hline S. number & Config-name & Simulated gain $(\mathrm{dBi})$ & Measured gain $(\mathrm{dBi})$ & Radiation efficiency\% \\
\hline$(1)$ & Config-I & 3.68 & 2.91 & 92 \\
$(2)$ & Config-II & 3.74 & 3.11 & 91 \\
$(3)$ & Config-III & 3.66 & 3.07 & 93 \\
$(4)$ & Config-IV & 5.02 & 4.73 & 92 \\
$(5)$ & Config-V & 6.05 & 5.45 & 93 \\
\hline
\end{tabular}

\section{Conflicts of Interest}

The authors declare that there are no conflicts of interest regarding the publication of this work.

\section{Acknowledgments}

The authors would like to thank the Ministry of Education (MOE) and UTM GUP (Vot 4J211, 05H62, 03G33, and 11H59) for sponsoring this work.

\section{References}

[1] S. W. Lee and Y. Sung, "Compact frequency reconfigurable antenna for LTE/WWAN mobile handset applications," Institute of Electrical and Electronics Engineers. Transactions on Antennas and Propagation, vol. 63, no. 10, pp. 4572-4577, 2015.

[2] J. Nasir, M. H. Jamaluddin, M. Khalily, M. R. Kamarudin, I. Ullah, and R. Selvaraju, "A reduced size dual port MIMO DRA with high isolation for $4 \mathrm{G}$ applications," International Journal of RF and Microwave Computer-Aided Engineering, vol. 25, no. 6, pp. 495-501, 2015.

[3] S. Danesh, S. K. A. Rahim, M. Abedian, M. Khalily, and M. R. Hamid, "Frequency-reconfigurable rectangular dielectric resonator antenna," IEEE Antennas and Wireless Propagation Letters, vol. 12, pp. 1331-1334, 2013.

[4] S. Aqeel, M. H. Jamaluddin, A. A. Khan et al., "A DualBand Multiple Input Multiple Output Frequency Agile Antenna for GPSL1/Wi-Fi/WLAN2400/LTE Applications," International Journal of Antennas and Propagation, vol. 2016, Article ID 9419183, 2016.

[5] H.-M. Chen, Y.-K. Wang, Y.-F. Lin, S.-C. Lin, and S.-C. Pan, "A compact dual-band dielectric resonator antenna using a parasitic slot," IEEE Antennas and Wireless Propagation Letters, vol. 8, pp. 173-176, 2009.

[6] M. A. Saed and R. Yadla, "Microstrip-fed low profile and compact dielectric resonator antennas," Progress in Electromagnetics Research, vol. 56, pp. 151-162, 2006.

[7] D. Cormos, A. Laisne, R. Gillard, F. Le Bolzer, and C. Nicolas, "Compact dielectric resonator antenna for WLAN applications," Electronics Letters, vol. 39, no. 7, pp. 588-590, 2003.

[8] Y. Ge, W. Tang, and H. Zhang, "Stacked compact dielectric resonator antennas for broadband applications," in Proceedings of the 2012 10th International Symposium on Antennas, Propagation and EM Theory, ISAPE 2012, pp. 117-120, chn, October 2012.

[9] T. A. Denidni and Q. Rao, "Hybrid dielectric resonator antennas with radiating slot for dual-frequency operation," IEEE Antennas and Wireless Propagation Letters, vol. 3, no. 1, pp. 321-323, 2004.
[10] Q. Rao, T. A. Denidni, A. R. Sebak, and R. H. Johnston, "Compact independent dual-band hybrid resonator antenna with multifunctional beams," IEEE Antennas and Wireless Propagation Letters, vol. 5, no. 1, pp. 239-242, 2006.

[11] Y. Gao, B.-L. Ooi, and A. P. Popov, "Dual-band hybrid dielectric resonator antenna with CPW-fed slot," Microwave and Optical Technology Letters, vol. 48, no. 1, pp. 170-172, 2006.

[12] X. S. Fang and K. W. Leung, "Designs of single-, dual-, wideband rectangular dielectric resonator antennas," IEEE Transactions on Antennas and Propagation, vol. 59, no. 6, pp. 2409-2414, 2011.

[13] A. Sharma and R. K. Gangwar, "Compact dual-band ring dielectric resonator antenna with moon-shaped defected ground structure for WiMAX/WLAN applications," International Journal of RF and Microwave Computer-Aided Engineering, vol. 26, no. 6, pp. 503-511, 2016.

[14] L. H. Weng, Y. Guo, X. Shi, and X. Chen, "An overview on defected ground structure," Progress In Electromagnetics Research B, vol. 7, pp. 173-189, 2008.

[15] A. Buerkle, K. Sarabandi, and H. Mosallaei, "Compact slot and dielectric resonator antenna with dual-resonance, broadband characteristics," IEEE Transactions on Antennas and Propagation, vol. 53, no. 3, pp. 1020-1027, 2005.

[16] M. H. Neshati and Z. Wu, "The determination of resonance frequency of the TE 111 mode in a rectangular dielectric resonator antenna, Proc," ICAPP, vol. 480, 2001.

[17] C. Caloz, H. Okabe, T. Iwai, and T. Itoh, "A Simple and Accurate Model for Microstrip Structures With Slotted Ground Plane," IEEE Microwave and Wireless Components Letters, vol. 14, no. 3, pp. 127-129, 2004.

[18] H. A. Wheeler, "Fundamentals limitations of small antennas," Proceding of the IRE, vol. 35, pp. 1479-1484, 1947. 


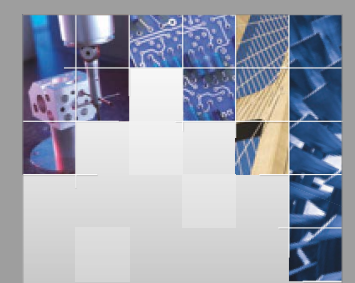

\section{Enfincering}
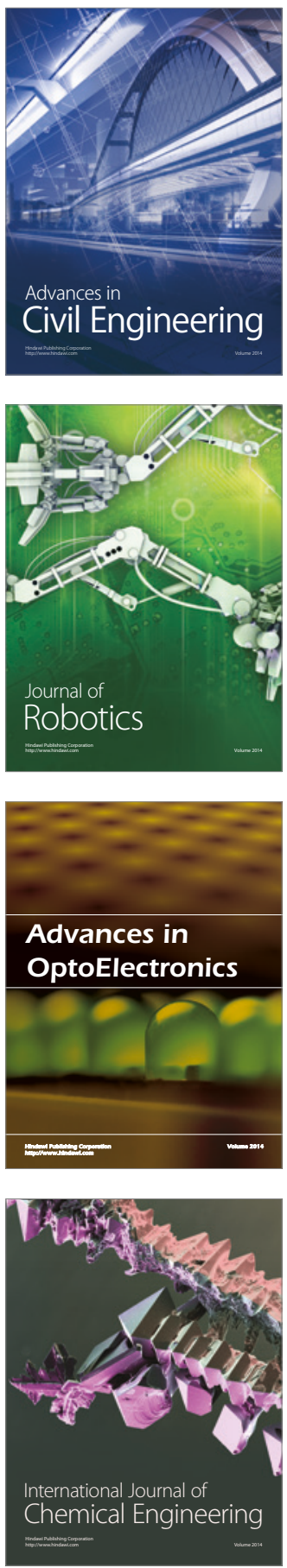

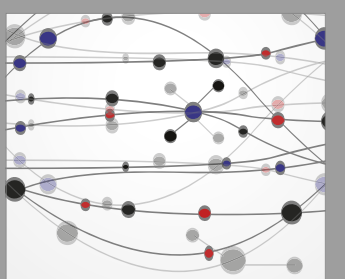

The Scientific World Journal

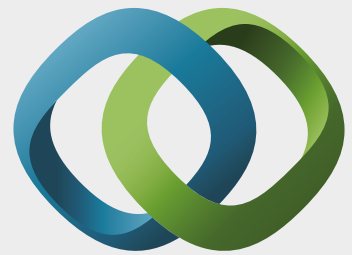

\section{Hindawi}

Submit your manuscripts at

https://www.hindawi.com
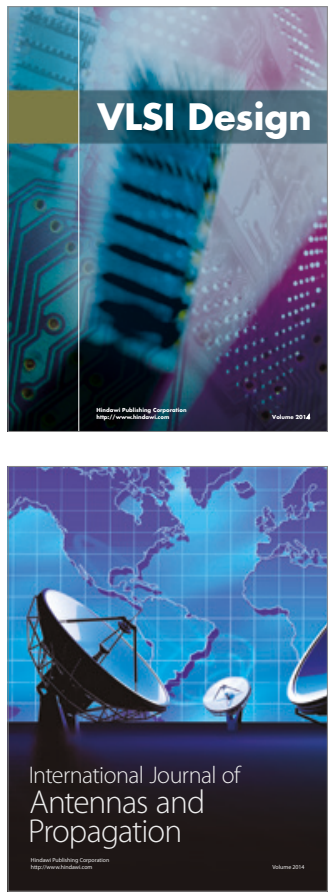

\section{Rotating}

Machinery
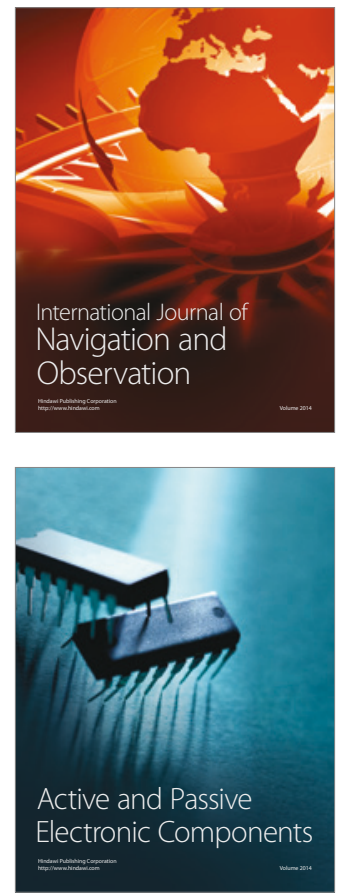
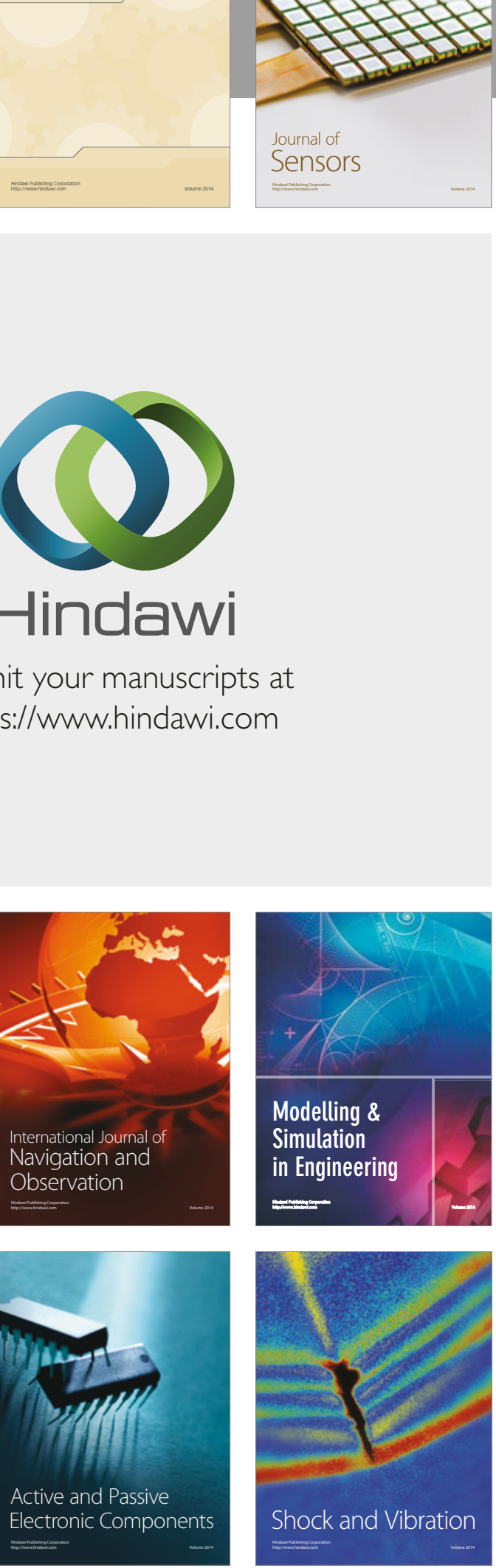
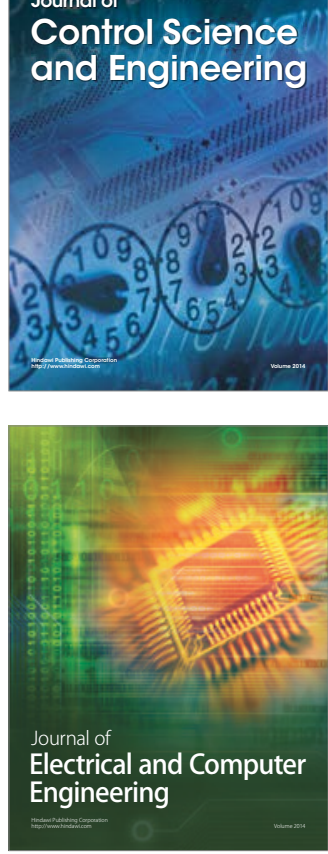

Distributed

Journal of

Control Science

and Engineering
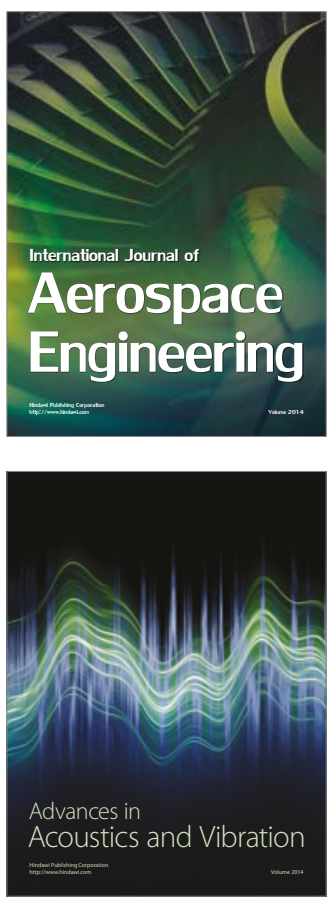

Sensor Networks 